\title{
Basic Forms and Orbit Spaces: a Diffeological Approach
}

\author{
Yael KARSHON ${ }^{\dagger}$ and Jordan WATTS W $^{\ddagger}$ \\ $\dagger$ Department of Mathematics, University of Toronto, \\ 40 St. George Street, Toronto Ontario M5S 2E4, Canada \\ E-mail: karshon@math.toronto.edu \\ URL: http://www.math.toronto.edu/karshon/ \\ $\ddagger$ Department of Mathematics, University of Colorado Boulder, \\ Campus Box 395, Boulder, CO, 80309, USA \\ E-mail: jordan.watts@colorado.edu \\ URL: http://euclid.colorado.edu/ jowa8403
}

Received October 06, 2015, in final form February 16, 2016; Published online March 08, 2016 http://dx.doi.org/10.3842/SIGMA.2016.026

\begin{abstract}
If a Lie group acts on a manifold freely and properly, pulling back by the quotient map gives an isomorphism between the differential forms on the quotient manifold and the basic differential forms upstairs. We show that this result remains true for actions that are not necessarily free nor proper, as long as the identity component acts properly, where on the quotient space we take differential forms in the diffeological sense.
\end{abstract}

Key words: diffeology; Lie group actions; orbit space; basic differential forms

2010 Mathematics Subject Classification: 58D19; 57R99

\section{Introduction}

Let $M$ be a smooth manifold and $G$ a Lie group acting on $M$. A basic differential form on $M$ is a differential form that is $G$-invariant and horizontal; the latter means that evaluating the form on any vector that is tangent to a $G$-orbit yields 0 . Basic differential forms constitute a subcomplex of the de Rham complex. If $G$ acts properly and with a constant orbit-type, then the quotient $M / G$ is a manifold, and, denoting the quotient map by $\pi: M \rightarrow M / G$, the pullback by this map gives an isomorphism of the de Rham complex on $M / G$ with the complex of basic forms on $M$. Even if $M / G$ is not a manifold, if $G$ acts properly, then the cohomology of the complex of basic forms is isomorphic to the singular cohomology of $M / G$ with real coefficients; this was shown by Koszul in 1953 [17] for compact group actions and by Palais in 1961 [19] for proper group actions. In light of these facts, some authors define the de Rham complex on $M / G$ to be the complex of basic forms on $M$.

There is another, intrinsic, definition of a differential form on $M / G$, which comes from viewing $M / G$ as a diffeological space (see Section 2). This definition agrees with the usual one when $M / G$ is a manifold. Differential forms on diffeological spaces admit exterior derivatives, wedge products, and pullbacks under smooth maps. Spaces of differential forms are themselves diffeological spaces too. With this notion, here is our main result:

Theorem 1.1. Let $G$ be a Lie group acting on a manifold $M$. Let $\pi: M \rightarrow M / G$ be the quotient map.

(i) The pullback map $\pi^{*}: \Omega^{*}(M / G) \rightarrow \Omega^{*}(M)$ is one-to-one. Its image is contained in the space $\Omega_{\text {basic }}^{*}(M)$ of basic forms. As a map to its image, the map $\pi^{*}$ is an isomorphism of differential graded algebras and a diffeological diffeomorphism. 
(ii) If the restriction of the action to the identity component of $G$ is proper, then the image of the pullback map is equal to the space of basic forms:

$$
\pi^{*}: \Omega^{*}(M / G) \stackrel{\cong}{\longrightarrow} \Omega_{\text {basic }}^{*}(M)
$$

We prove Theorem 1.1 in Section 5, after the proof of Proposition 5.10.

\section{Remark 1.2.}

1. Part (i) of Theorem 1.1, which follows from the results of Sections 2 and 3, is not difficult. The technical heart of Theorem 1.1 is Part (ii), which is proved in Proposition 5.10: if the identity component of the group acts properly, then every basic form on $M$ descends to a diffeological form on $M / G$. This fact is non-trivial even when the group $G$ is finite.

2. The quotient $M / G$ can be non-Hausdorff. Nevertheless, even if its topology is trivial, $M / G$ may have non-trivial differential forms, and its de Rham cohomology may be non-trivial. See, for example, the irrational torus in Remark 5.12.

3. We expect the conclusion of Part (ii) of Theorem 1.1 to hold under more general hypotheses. In particular, our assumption that the identity component of $G$ act properly on $M$ is sufficient but not necessary; see, for instance, Example 5.13.

Diffeology was developed by Jean-Marie Souriau (see [26]) around 1980, following earlier work of Kuo-Tsai Chen (see, e.g., [4, 5]). Our primary reference for this theory is the book [14] by Iglesias-Zemmour. In many applications, diffeology can serve as a replacement for the manifold structures (modelled on locally convex topological vector spaces) on spaces of smooth paths, functions, or differential forms. See, for example, [2].

The category of diffeological spaces is complete and co-complete (see [1]); in particular, subsets and quotients naturally inherit diffeological structures. It is also Cartesian closed, where we equip spaces of smooth maps with their natural functional diffeology.

It is also common to consider $M / G$ as a (Sikorski) differential space, by equipping it with the set of those real valued functions whose pullback to $M$ is smooth. See Hochschild [12], Bredon [3], G. Schwarz [22], and Cushman and Śniatycki [7]. This structure is determined by the diffeology on $M / G$ but is weaker. For example, the quotients $\mathbb{R}^{n} / \mathrm{SO}(n)$ for different positive integers $n$ are isomorphic as differential spaces but not as diffeological spaces. (See Exercise 50 of Iglesias [14] with solution at the end of the book.) There are several inequivalent notions of "differential form" on differential spaces (see Śniatycki [25] and Watts [27]); we do not know of an analogue of Theorem 1.1 for any of these notions.

Turning to higher category theory, we can also consider the stack quotient $[M / G]$. It is a differentiable stack over the site of manifolds, and it is represented by the action groupoid $G \times M \rightrightarrows M$. One can define a differential $k$-form on the stack $[M / G]$ as a map of stacks from $[M / G]$ to the stack of differential forms $\Omega^{k}$. In [30], Watts and Wolbert define a functor Coarse from stacks to diffeological spaces for which Coarse $([M / G])$ is equal to $M / G$ equipped with the quotient diffeology. In this language, Theorem 1.1 gives an isomorphism

$$
\Omega^{k}([M / G]) \cong \Omega^{k}(\operatorname{Coarse}([M / G])),
$$

when the identity component of $G$ acts properly. We note that a diffeological space can also be viewed as a stack, and applying Coarse recovers the original diffeological space. We also note that the quotient stack $[M / G]$ often contains more information than the quotient diffeology; for example, if $M$ is a point, the quotient diffeological space is a point, but the stack $[M / G]$ determines the group $G$. Finally, we note that Karshon and Zoghi [16] give sufficient conditions 
for a Lie groupoid to be determined up to Morita equivalence by its underlying diffeological space.

In the special case that the Lie group $G$ is compact, the main results of this paper appeared in the Ph.D. Thesis of the second author [28], supervised by the first author. A generalisation to proper Lie groupoids, which relies on these results and on a deep theorem of Crainic and Struchiner [6] showing that all proper Lie groupoids are linearisable, was worked out by Watts in [29].

The paper is structured as follows. For the convenience of the reader, Section 2 contains background on diffeology, differential forms on diffeological spaces, and Lie group actions in connection to diffeology. Section 3 contains a proof that the pullback map from the space of differential forms on the orbit space $M / G$ to the space of differential forms on the manifold $M$ is an injection into the space of basic forms, and is a diffeomorphism onto its image. Section 4 contains a technical lemma: "quotient in stages". Section 5 is the technical heart of the paper; it contains a proof that, if the identity component of the group acts properly, then the pullback map surjects onto the space of basic forms.

Our appendices contain two applications of the special case of Theorem 1.1 when the group $G$ is finite. In Appendix A we show that, on an orbifold, the notion of a diffeological differential form agrees with the usual notion of a differential form on the orbifold. In Appendix B we show that, on a regular symplectic quotient (which is also an orbifold), the notion of a diffeological differential form also agrees with Sjamaar's notion of a differential form on the symplectic quotient. The case of non-regular symplectic quotients is open.

\section{Background on diffeological spaces}

In this section we review the basics of diffeology, diffeological differential forms, and Lie group actions in the context of diffeology. For more details (e.g., the quotient diffeology is in fact a diffeology), see Iglesias-Zemmour [14].

\section{The basics of diffeology}

This subsection contains a review of the basics of diffeology; in particular, the definition of a diffeology and diffeologically smooth maps, as well as various constructions in the diffeological category.

Definition 2.1 (diffeology). Let $X$ be a set. A parametrisation on $X$ is a function $p: U \rightarrow X$ where $U$ is an open subset of $\mathbb{R}^{n}$ for some $n$. A diffeology $\mathcal{D}$ on $X$ is a set of parametrisations that satisfies the following three conditions.

1. (Covering) For every point $x \in X$ and every non-negative integer $n \in \mathbb{N}$, the constant function $p: \mathbb{R}^{n} \rightarrow\{x\} \subseteq X$ is in $\mathcal{D}$.

2. (Locality) Let $p: U \rightarrow X$ be a parametrisation such that for every point in $U$ there exists an open neighbourhood $V$ in $U$ such that $\left.p\right|_{V} \in \mathcal{D}$. Then $p \in \mathcal{D}$.

3. (Smooth compatibility) Let $(p: U \rightarrow X) \in \mathcal{D}$. Then for every $n \in \mathbb{N}$, every open subset $V \subseteq \mathbb{R}^{n}$, and every smooth map $F: V \rightarrow U$, we have $p \circ F \in \mathcal{D}$.

A set $X$ equipped with a diffeology $\mathcal{D}$ is called a diffeological space and is denoted by $(X, \mathcal{D})$. When the diffeology is understood, we drop the symbol $\mathcal{D}$. The elements of $\mathcal{D}$ are called plots.

Example 2.2 (standard diffeology on a manifold). Let $M$ be a manifold. The standard diffeology on $M$ is the set of all smooth maps to $M$ from open subsets of $\mathbb{R}^{n}$ for all $n \in \mathbb{N}$. 
Definition 2.3 (diffeologically smooth maps). Let $X$ and $Y$ be two diffeological spaces, and let $F: X \rightarrow Y$ be a map. We say that $F$ is (diffeologically) smooth if for any plot $p: U \rightarrow X$ of $X$ the composition $F \circ p: U \rightarrow Y$ is a plot of $Y$. Denote by $C^{\infty}(X, Y)$ the set of all smooth maps from $X$ to $Y$. Denote by $C^{\infty}(X)$ the set of all smooth maps from $X$ to $\mathbb{R}$, where $\mathbb{R}$ is equipped with its standard diffeology.

Remark 2.4 (plots). A parametrisation is diffeologically smooth if and only if it is a plot.

Remark 2.5 (smooth maps between manifolds). A map between two manifolds is diffeologically smooth if and only if it is smooth in the usual sense. In particular, if $M$ is a manifold then $C^{\infty}(M)$ is the usual set of smooth real valued functions.

Remark 2.6. Diffeological spaces, along with diffeologically smooth maps, form a category. It is shown in [1, Theorem 3.2] that this category is a complete and cocomplete quasi-topos. In particular, it is closed under passing to arbitrary quotients, subsets, function spaces, products, and coproducts.

Definition 2.7 (quotient diffeology). Let $X$ be a diffeological space, and let $\sim$ be an equivalence relation on $X$. Let $Y=X / \sim$ be the quotient set, and let $\pi: X \rightarrow Y$ be the quotient map. We define the quotient diffeology on $Y$ to be the diffeology for which the plots are those maps $p: U \rightarrow Y$ such that for every point in $U$ there exist an open neighbourhood $V \subseteq U$ and a plot $q: V \rightarrow X$ such that $\left.p\right|_{V}=\pi \circ q$.

Remark 2.8 (quotient map). Let $X$ be a diffeological space and $\sim$ an equivalence relation on $X$. Then the quotient map $\pi: X \rightarrow X / \sim$ is smooth.

A special case that is important to us is the quotient of a manifold by the action of a Lie group.

Definition 2.9 (subset diffeology). Let $X$ be a diffeological space, and let $Y$ be a subset of $X$. The subset diffeology on $Y$ consists of those maps to $Y$ whose composition with the inclusion map $Y \rightarrow X$ are plots of $X$.

Definition 2.10 (product diffeology). Let $X$ and $Y$ be two diffeological spaces. The product diffeology on the set $X \times Y$ is defined as follows. Let $\operatorname{pr}_{X}: X \times Y \rightarrow X$ and $\operatorname{pr}_{Y}: X \times Y \rightarrow Y$ be the natural projections. A parametrisation $p: U \rightarrow X \times Y$ is a plot if $\operatorname{pr}_{X} \circ p$ and $\operatorname{pr}_{Y} \circ p$ are plots of $X$ and $Y$, respectively.

Definition 2.11 (standard functional diffeology on maps). Let $Y$ and $Z$ be diffeological spaces. The standard functional diffeology on $C^{\infty}(Y, Z)$ is defined as follows. A parametrisation $p: U \rightarrow$ $C^{\infty}(Y, Z)$ is a plot if the map

$$
U \times Y \rightarrow Z \quad \text { given by } \quad(u, y) \mapsto p(u)(y)
$$

is smooth.

\section{Diffeological differential forms}

In this subsection we review differential forms on diffeological spaces, as well as introduce Proposition 2.20 which is a simple criterion crucial to the proof of Theorem 1.1.

Definition 2.12 (differential forms). Let $(X, \mathcal{D})$ be a diffeological space. A (diffeological) differential $k$-form $\alpha$ on $X$ is an assignment to each plot $(p: U \rightarrow X) \in \mathcal{D}$ a differential $k$-form $\alpha(p) \in \Omega^{k}(U)$ satisfying the following smooth compatibility condition: for every open subset $V$ of a Euclidean space and every smooth map $F: V \rightarrow U$,

$$
\alpha(p \circ F)=F^{*}(\alpha(p)) .
$$

Denote the set of differential $k$-forms on $X$ by $\Omega^{k}(X)$. 
Definition 2.13 (pullback map). Let $X$ and $Y$ be diffeological spaces, and let $F: X \rightarrow Y$ be a diffeologically smooth map. Let $\alpha$ be a differential $k$-form on $Y$. Define the pullback $F^{*} \alpha$ to be the $k$-form on $X$ that satisfies the following condition: for every plot $p: U \rightarrow X$,

$$
F^{*} \alpha(p)=\alpha(F \circ p) .
$$

Example 2.14. Let $\alpha$ be a differential form on a manifold $M$. Then $(p: U \rightarrow M) \mapsto p^{*} \alpha$ defines a diffeological differential form on $M$. In this way, we get an identification of the ordinary differential forms on $M$ with the diffeological differential forms on $M$.

Let $X$ be a diffeological space, $\alpha$ a differential form on $X$, and $p: U \rightarrow X$ a plot. The above identification of ordinary differential forms on $U$ with diffeological differential forms on $U$ gives $\alpha(p)=p^{*} \alpha$. Henceforth, we may write $p^{*} \alpha$ instead of $\alpha(p)$.

Example 2.15. The space $\Omega^{0}(X)$ of diffeological 0-forms is identified with the space $C^{\infty}(X)$ of smooth real valued functions, by identifying the function $f$ with the 0 -form $(p: U \rightarrow X) \mapsto f \circ p$. See [14, Section 6.31]. With this identification, the pullback of 0-forms by a smooth map $F$ becomes the precomposition of smooth real-valued functions by $F$.

Remark 2.16 (pullback is linear). The space of differential forms on a diffeological space is naturally a linear vector space: for $\alpha, \beta \in \Omega^{k}(X)$ and $a, b \in \mathbb{R}$, we define $a \alpha+b \beta:(p: U \rightarrow$ $X) \mapsto a \alpha(p)+b \beta(p)$. If $F: X \rightarrow Y$ is a smooth map of diffeological spaces, then the pullback $\operatorname{map} F^{*}: \Omega^{k}(Y) \rightarrow \Omega^{k}(X)$ is linear.

Remark 2.17 (wedge product and exterior derivative). Let $X$ be a diffeological space. Define the wedge product of $\alpha \in \Omega^{k}(X)$ and $\beta \in \Omega^{l}(X)$ to be the $(k+l)$-form $\alpha \wedge \beta:(p: U \rightarrow X) \mapsto$ $p^{*} \alpha \wedge p^{*} \beta$. Define the exterior derivative of $\alpha$ to be the $k+1$ form $d \alpha:(p: U \rightarrow X) \mapsto d\left(p^{*} \alpha\right)$. Then $\Omega^{*}(X)=\bigoplus_{k=0}^{\infty} \Omega^{k}(X)$ is a differential graded algebra. In particular, $\Omega^{*}(X)$ is an exterior algebra, and $\left(\Omega^{*}(X), d\right)$ is a complex. If $F: X \rightarrow Y$ is a smooth map of diffeological spaces, then the pullback map $F^{*}: \Omega^{*}(Y) \rightarrow \Omega^{*}(X)$ is a morphism of differential graded algebras; in particular, it intertwines the wedge products and the exterior derivatives.

Definition 2.18 (standard functional diffeology on forms). Let $(X, \mathcal{D})$ be a diffeological space. The standard functional diffeology on $\Omega^{k}(X)$ is defined as follows. A parametrisation $p: U \rightarrow$ $\Omega^{k}(X)$ is a plot if for every plot $(q: V \rightarrow X) \in \mathcal{D}$, where $V$ is open in $\mathbb{R}^{n}$, the map $U \times V \rightarrow \bigwedge^{k} \mathbb{R}^{n}$ sending $(u, v)$ to $\left.q^{*}(p(u))\right|_{v}$ is smooth. See [14, Section 6.29] for a proof that this is indeed a diffeology.

Remark 2.19. Let $X$ be a diffeological space. We have the following facts.

1. Under the identification of Example 2.15 of the space of diffeological 0-forms with the space of smooth real valued functions, Definitions 2.11 and 2.18 of the standard functional diffeology on these spaces agree.

2. If $F: X \rightarrow Y$ is a smooth map to another diffeological space, then the pullback map $F^{*}: \Omega^{k}(Y) \rightarrow \Omega^{k}(X)$ is smooth with respect to the standard functional diffeologies on the sets of differential forms. See [14, Section 6.32].

3. The exterior derivative $d: \Omega^{k}(X) \rightarrow \Omega^{k+1}(X)$ and the wedge product $\Omega^{k}(X) \times \Omega^{l}(X) \rightarrow$ $\Omega^{k+l}(X)$ are smooth. See Sections 6.34 and 6.35 of [14].

Proposition 2.20 (pullbacks of quotient diffeological forms). Let $G$ be a Lie group, acting on a manifold $M$, and let $\pi: M \rightarrow M / G$ be the quotient map. Then a differential form $\alpha$ on $M$ is in the image of $\pi^{*}$ if and only if, for every two plots $p_{1}: U \rightarrow M$ and $p_{2}: U \rightarrow M$ such that $\pi \circ p_{1}=\pi \circ p_{2}$, we have

$$
p_{1}^{*} \alpha=p_{2}^{*} \alpha \text {. }
$$

Proof. This result is a special case of [14, Section 6.38]. 


\section{Group actions}

Here we highlight some facts about Lie group actions in connection to diffeology, as well as review the definition of basic forms. Finally, we introduce another crucial ingredient to the proof of Theorem 1.1, the slice theorem (Theorem 2.25). Koszul [17] proved the slice theorem for compact Lie group actions, and Palais [19] proved it for proper Lie group actions. The proof is also described in Theorem 2.3.3 of [9] and in Appendix B of [10].

Lemma 2.21. Let a Lie group $G$ act on a manifold $M$. Let $x$ be a point in $M$ and $H$ its stabiliser. Then

- There exists a unique manifold structure on the quotient $G / H$ such that the quotient map $G \rightarrow G / H$ is a submersion. The standard diffeology on this manifold agrees with the quotient diffeology induced from $G$.

- There exists a unique manifold structure on the orbit $G \cdot x$ such that the inclusion map $G \cdot x \rightarrow M$ is an immersion. The standard diffeology on this manifold agrees with the subset diffeology induced from $M$.

- The orbit map $a \mapsto a \cdot x$ from $G$ to $M$ descends to a diffeomorphism from $G / H$ to $G \cdot x$.

- The tangent space $T_{x}(G \cdot x)$ is the space of vectors $\left.\xi_{M}\right|_{x}$ for $\xi \in \mathfrak{g}$, where $\xi_{M}$ is the vector field on $M$ that is induced by the Lie algebra element $\xi$. This space is also the image of the differential at the identity of the orbit map $a \mapsto a \cdot x$ from $G$ to $M$.

Proof. See [15, Section 2, Paragraph 1].

Definition 2.22 (basic forms). Let $G$ be a Lie group acting on a manifold $M$. A differential form $\alpha$ on $M$ is horizontal if for any $x \in M$ and $v \in T_{x}(G \cdot x)$ we have

$$
v\lrcorner \alpha=0 .
$$

(Recall that $v\lrcorner \alpha=\alpha(v, \cdot, \ldots, \cdot)$.) A form that is both horizontal and $G$-invariant is called basic. When the $G$-action is understood, we denote the set of basic $k$-forms on $M$ by $\Omega_{\text {basic }}^{k}(M)$.

Remark 2.23. The space of basic differential forms on a $G$-manifold $M$ is closed under linear combinations, wedge products, and exterior derivatives.

Remark 2.24. Given a quotient map $\pi: X \rightarrow X^{\prime}$ of diffeological spaces, (more generally, given a so-called subduction,) Iglesias-Zemmour [14, Section 6.38] defines a "basic form" to be a differential form on $X$ that satisfies the technical condition that appears in Proposition 2.20 above. Our results show that, for smooth Lie group actions where the identity component acts properly, Iglesias-Zemmour's definition agrees with the usual one.

Let $G$ be a Lie group, $H$ a closed subgroup, and $V$ a vector space with a linear $H$-action. The equivariant vector bundle

$$
G \times{ }_{H} V
$$

over $G / H$ is obtained as the quotient of $G \times V$ by the anti-diagonal $H$-action $h \cdot(g, v)=$ $\left(g h^{-1}, h \cdot v\right)$. The $G$-action on $G \times_{H} V$ is $g \cdot\left[g^{\prime}, v\right]=\left[g g^{\prime}, v\right]$.

Theorem 2.25 (slice theorem). Let $G$ be a Lie group acting properly on a manifold $M$. Fix $x \in M$. Let $H$ be the stabiliser of $x$, and let $V=T_{x} M / T_{x}(G \cdot x)$ be the normal space to the orbit $G \cdot x$ at $x$, equipped with the linear $H$-action that is induced by the linear isotropy action of $H$ on $T_{x} M$. Then there exist a $G$-invariant open neighbourhood $U$ of $x$ and a $G$-equivariant diffeomorphism $F: U \rightarrow G \times_{H} V$ that takes $x$ to $[1,0]$. 


\section{The pullback injects into basic forms}

In this section we prove the easy part of Theorem 1.1: for a Lie group $G$ acting on a manifold $M$ with quotient map $\pi: M \rightarrow M / G$, the pullback map $\pi^{*}$ is an injection from the set of diffeological differential forms on $M / G$ into the set of basic forms on $M$, and $\pi^{*}$ is a diffeomorphism onto its image.

The following lemma is a special case of [14, Section 6.39].

Lemma 3.1. Let a Lie group $G$ act on a manifold $M$, and let $\pi: M \rightarrow M / G$ be the quotient map. Then the pullback map on forms, $\pi^{*}: \Omega^{k}(M / G) \rightarrow \Omega^{k}(M)$, is an injection.

Proof. More generally, let $X$ be a diffeological space, $\sim$ an equivalence relation on $X$, and $\pi: X \rightarrow X / \sim$ the quotient map. Then the pullback map on forms, $\pi^{*}: \Omega^{k}(X / \sim) \rightarrow \Omega^{k}(X)$, is an injection.

By Remark 2.16 it is enough to show that the kernel of the pullback map

$$
\pi^{*}: \Omega^{k}(X / \sim) \rightarrow \Omega^{k}(X)
$$

is trivial. Let $\alpha \in \Omega^{k}(X / \sim)$ be such that $\pi^{*} \alpha=0$. Then, for any plot $p: U \rightarrow X$, we have $p^{*} \pi^{*} \alpha=0$. By the definition of the quotient diffeology, this implies that for any plot $q: U \rightarrow X / \sim$ we have $q^{*} \alpha=0$. Hence, $\alpha=0$, as required.

Proposition 3.2 (pullbacks from the orbit space are basic). Let a Lie group $G$ act on a manifold $M$. Let $\alpha=\pi^{*} \beta$ for some $\beta \in \Omega^{k}(M / G)$. Then $\alpha$ is basic.

Proof. To show that $\alpha$ is $G$-invariant, note that for every $g \in G$, because $\pi \circ g=\pi$,

$$
g^{*} \alpha=g^{*} \pi^{*} \beta=\pi^{*} \beta=\alpha .
$$

If $\alpha$ is a zero-form (that is, a smooth function) then $\alpha$ is automatically horizontal and we are done. Next, we assume that $\alpha$ is a differential form of positive degree, and we show that $\alpha$ is horizontal. By Lemma 2.21, if $x \in M$ and $v \in T_{x}(G \cdot x)$, then there exists $\xi \in \mathfrak{g}$ such that

$$
v=\left.\frac{d}{d t}\right|_{t=0} \exp (t \xi) \cdot x
$$

Let $A_{x}: G \rightarrow M$ be the map sending $g$ to $g \cdot x$. Then, $v=\left(A_{x}\right)_{*}(\xi)$.

Thus, to show that $\alpha$ is horizontal, it is enough to show that $A_{x}^{*} \alpha=0$ for all $x \in M$. Indeed, it follows from the following commutative diagram that $A_{x}^{*} \alpha=A_{x}^{*} \pi^{*} \beta=0$,

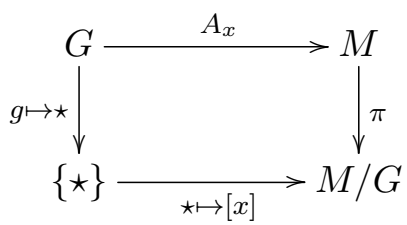

Remark 3.3. Proposition 3.2 can also be deduced from the following lemma: a differential form $\alpha$ on $M$ is basic if and only if its pullbacks under the maps $G \times M \rightarrow M$ given by the projection $(g, x) \mapsto x$ and by the action $(g, x) \mapsto g \cdot x$ coincide. For a proof of this lemma see, for example, [29, Lemma 3.3].

Proposition 3.4 (pullbacks via quotient maps). Let a Lie group $G$ act on a manifold $M$, and let $\pi: M \rightarrow M / G$ be the quotient map. Then the pullback map

$$
\pi^{*}: \Omega^{k}(M / G) \rightarrow \pi^{*} \Omega^{k}(M / G)
$$

is a diffeomorphism, where the target space is equipped with the subset diffeology induced from $\Omega^{k}(M)$. 
Proof. More generally, let $X$ be a diffeological space, let $\sim$ be an equivalence relation on $X$, and let $\pi: X \rightarrow X / \sim$ be the quotient map. We will show that the pullback map

$$
\pi^{*}: \Omega^{k}(X / \sim) \rightarrow \pi^{*} \Omega^{k}(X / \sim)
$$

is a diffeomorphism, where the target space is equipped with the subset diffeology induced from $\Omega^{k}(X)$.

Clearly, $\pi^{*}$ is surjective to its image. For the injectivity of $\pi^{*}$, see Lemma 3.1. As noted in Part (2) of Remark $2.19, \pi^{*}$ is smooth. We wish to show that the inverse map

$$
\left(\pi^{*}\right)^{-1}: \pi^{*} \Omega^{k}(X / \sim) \rightarrow \Omega^{k}(X / \sim)
$$

is also smooth.

Fix a plot $p: U \rightarrow \Omega^{k}(X)$ with image in $\pi^{*} \Omega^{k}(X / \sim)$. We would like to show that $\left(\pi^{*}\right)^{-1} \circ$ $p: U \rightarrow \Omega^{k}(X / \sim)$ is a plot of $\Omega^{k}(X / \sim)$. By Definition 2.18 of the diffeology on spaces of differential forms, we need to show, given any plot $r: W \rightarrow X / \sim$ with $W \subset \mathbb{R}^{n}$, that

$$
\left.(u, w) \mapsto r^{*}\left(\left(\left(\pi^{*}\right)^{-1} \circ p\right)(u)\right)\right|_{w}
$$

is a map to $\bigwedge^{k} \mathbb{R}^{n}$ that is smooth in $(u, w) \in U \times W$. Here, $\left(\pi^{*}\right)^{-1}$ is restricted to the image of $\pi^{*}$, on which it is well defined because $\pi^{*}$ is injective.

It is enough to show smoothness locally. For any point $w \in W$ there exist an open neighbourhood $V \subseteq W$ of $w$ and a plot $q: V \rightarrow X$ such that $\left.r\right|_{V}=\pi \circ q$. For all $v \in V$, we have

$$
\left.r^{*}\left(\left(\left(\pi^{*}\right)^{-1} \circ p\right)(u)\right)\right|_{v}=\left.q^{*}(p(u))\right|_{v}
$$

which is smooth in $(u, v) \in U \times V$ by the definition of the standard functional diffeology on $\Omega^{k}(X)$. And so we are done.

\section{Quotient in stages}

In this section we give a technical result that we use in the next section. All quotients, subsets, and products are assumed to be equipped with the quotient, subset, and product diffeologies.

On the image of any Lie group homomorphism $H \rightarrow G$ there exists a unique manifold structure such that the inclusion map of the image into $G$ is an immersion; this follows from the second item of Lemma 2.21. By Lie subgroup of $G$ we refer to such an image. Thus, Lie subgroups are injectively immersed subgroups that are not necessarily closed.

Recall that a Lie group $G$ acts properly on a manifold $N$ if the map $G \times N \rightarrow N \times N$ sending $(g, x)$ to $(x, g \cdot x)$ is proper. The action is said to have constant orbit-type if all stabilisers are conjugate. If a Lie group acts properly and with a constant orbit type, then the quotient is a manifold and the quotient map is a fibre bundle.

Lemma 4.1 (quotient in stages). Let a Lie group $G$ act on a manifold $N$. Let $K$ be a Lie subgroup of $G$ that is normal in $G$. Also consider the induced action of $G$ on the quotient $N / K$.

(i) There exists a unique map $e: N / G \rightarrow(N / K) / G$ such that the following diagram commutes:

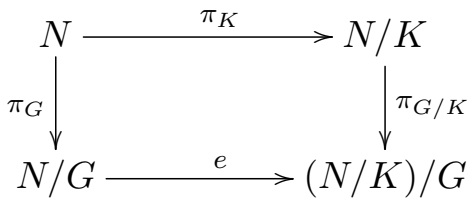


(ii) The map e is a diffeomorphism.

(iii) The pullback map

$$
\pi_{K}^{*}: \Omega^{*}(N / K) \rightarrow \Omega^{*}(N)
$$

restricts to a bijection from Image $\pi_{G / K}^{*}$ onto Image $\pi_{G}^{*}$.

(iv) Suppose that $K$ acts on $N$ properly and with a constant orbit-type, so that $N / K$ is a manifold and $N \rightarrow N / K$ is a fibre bundle. Then the pullback map $\pi_{K}^{*}$ also restricts to a bijection from $\Omega_{\text {basic }}^{*}(N / K)$ onto $\Omega_{\text {basic }}^{*}(N)$. Consequently, if one of the inclusions Image $\pi_{G / K}^{*} \subset$ $\Omega_{\text {basic }}^{*}(N / K)$ and Image $\pi_{G}^{*} \subset \Omega_{\text {basic }}^{*}(N)$ of Proposition 3.2 is an equality, then so is the other.

Remark 4.2. The $G$-action on $N / K$ factors through an action of $G / K$. The quotients $(N / K) / G$ and $(N / K) /(G / K)$ coincide, as they are quotients of $N / K$ by the same equivalence relation. If $K$ is closed in $G$ (so that $G / K$ is a Lie group) and $N / K$ is a manifold, then $G$-basic forms coincide with $(G / K)$-basic forms on $N / K$.

Proof of Lemma 4.1. Because $\pi_{K}$ is $G$-equivariant, such a map e exists. Because $\pi_{G}$ is onto, such a map $e$ is unique. Because the preimage under $\pi_{K}$ of a $G$-orbit in $N / K$ is a single $G$-orbit in $N$, the map $e$ is one-to-one. Because the maps $\pi_{G / K}$ and $\pi_{K}$ are onto, the map $e$ is onto.

Thus, the map $e$ is a bijection. To show that it is a diffeomorphism, it remains to show, for every parametrisation $p: U \rightarrow N / G$, that $p$ is a plot of $N / G$ if and only if $e \circ p$ is a plot of $(N / K) / G$.

Fix a parametrisation,

$$
p: \quad U \rightarrow N / G .
$$

Suppose that $p$ is a plot of $N / G$. Let $u \in U$. Then there exist an open neighbourhood $W$ of $u$ in $U$ and a plot $q: W \rightarrow N$ such that $\left.p\right|_{W}=\pi_{G} \circ q$. The composition $\pi_{K} \circ q$ is a plot of $N / K$, and

$$
\pi_{G / K} \circ \pi_{K} \circ q=e \circ \pi_{G} \circ q=\left.e \circ p\right|_{W} .
$$

Because $u \in U$ was arbitrary, this shows that $e \circ p$ is a plot of $(N / K) / G$.

Conversely, suppose that $e \circ p$ is a plot of $(N / K) / G$. Let $u \in U$. By applying the definition of the quotient diffeology at $\pi_{G / K}$ and then at $\pi_{K}$, we obtain an open neighbourhood $W$ of $u$ in $U$ and a plot $r: W \rightarrow N$ such that

$$
\left.e \circ p\right|_{W}=\pi_{G / K} \circ \pi_{K} \circ r .
$$

By (4.1) and by the choice of $r$,

$$
e \circ \pi_{G} \circ r=\pi_{G / K} \circ \pi_{K} \circ r=\left.e \circ p\right|_{W} .
$$

Because $e$ is one-to-one, this implies that $\pi_{G} \circ r=\left.p\right|_{W}$. Because $u \in U$ was arbitrary, this shows that $p$ is a plot of $N / G$. This completes the proof that $e$ is a diffeomorphism.

Because the diagram (4.1) commutes, $\pi_{K}^{*}$ takes Image $\pi_{G / K}^{*}$ into Image $\pi_{G}^{*}$. Because $e$ is a diffeomorphism, we can consider its inverse. From the commuting diagram

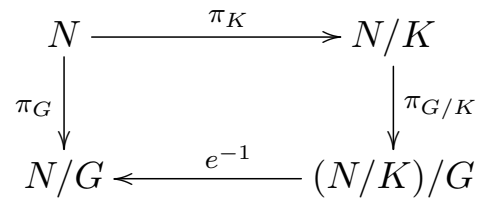


we see that $\pi_{K}^{*}$ takes Image $\pi_{G / K}^{*}$ onto Image $\pi_{G}^{*}$. Because $\pi_{K}^{*}$ is one-to-one (by Lemma 3.1), we have a bijection $\pi_{K}^{*}$ : Image $\pi_{G / K}^{*} \rightarrow$ Image $\pi_{G}^{*}$.

Now suppose that $K$ acts on $N$ properly and with a constant orbit-type, so that $N / K$ is a manifold and $\pi_{K}$ is a fibre bundle. Then we know that $\pi_{K}^{*}$ is a bijection from the differential forms on $N / K$ to the $K$-basic differential forms on $N$.

Because $\pi_{K}$ is $G$-equivariant, $\pi_{K}^{*}$ takes $G$-invariant forms on $N / K$ to $G$-invariant forms on $N$ and $G$-horizontal forms on $N / K$ to $G$-horizontal forms on $N$. So we have an injection $\pi_{K}^{*}: \Omega_{\text {basic }}^{*}(N / K) \rightarrow \Omega_{\text {basic }}^{*}(N)$.

Let $\alpha$ be a $G$-basic form on $N$. In particular $\alpha$ is $K$-basic, so there exists a form $\beta$ on $N / K$ such that $\alpha=\pi_{K}^{*} \beta$. Because $\pi_{K}^{*}$ is one-to-one and $\alpha$ is $G$-invariant, $\beta$ is $G$-invariant. Because $\pi_{K}$ is $G$-equivariant and $\alpha$ is $G$-horizontal, $\beta$ is $G$-horizontal. This completes the proof that the $\operatorname{map} \pi_{K}^{*}: \Omega_{\text {basic }}^{*}(N / K) \rightarrow \Omega_{\text {basic }}^{*}(N)$ is a bijection.

\section{The pullback surjects onto basics forms}

The main result of this section is Proposition 5.10, in which we give conditions on an action of a Lie group $G$ on a manifold $M$ under which every basic form $\alpha$ is the pullback of some diffeological form on the quotient. By Proposition 2.20, we need to show that $p_{1}^{*} \alpha=p_{2}^{*} \alpha$ for every two plots $p_{1}: U \rightarrow M$ and $p_{2}: U \rightarrow M$ such that for each $u \in U$ there is some $g \in G$ such that $p_{2}(u)=g \cdot p_{1}(u)$. If $g$ can be chosen to be a smooth function of $u$, then it is easy to conclude that $p_{1}^{*} \alpha=p_{2}^{*} \alpha$ if $\alpha$ is basic:

Lemma 5.1. Let a Lie group $G$ act on a manifold $M$. Let $p_{1}, p_{2}: U \rightarrow M$ be plots. Suppose that $p_{2}(u)=a(u) \cdot p_{1}(u)$ for some smooth function $a: U \rightarrow G$. Then for every $\alpha \in \Omega_{\text {basic }}^{k}(M)$ we have $p_{1}^{*} \alpha=p_{2}^{*} \alpha$.

Proof. Pick a point $u \in U$ and a tangent vector $v \in T_{u} U$. Let $\xi_{1}=\left(p_{1}\right)_{*} v\left(\in T_{p_{1}(u)} M\right)$ and $\xi_{2}=\left(p_{2}\right)_{*} v\left(\in T_{p_{2}(u)} M\right)$. Let $g=a(u)$. The directional derivative $\left.D_{v} a\right|_{u}$ of $a(\cdot)$ in the direction of $v$ has the form $\eta \cdot g\left(\in T_{g} G\right)$ for some Lie algebra element $\eta$ (i.e., it is the right translation of $\eta$ by $g$ ). We then have that

$$
\xi_{2}=g \cdot \xi_{1}+\left.\eta_{M}\right|_{p_{2}(u)}
$$

where $g \cdot \xi_{1}$ is the image of $\xi_{1}$ under the differential (push-forward) map $g_{*}: T_{p_{1}(u)} M \rightarrow T_{p_{2}(u)} M$, and where $\eta_{M}$ is the vector field on $M$ that corresponds to $\eta$; in particular $\eta_{M}$ is everywhere tangent to the $G$ orbits.

Applying this to vectors $v^{(1)}, \ldots, v^{(k)} \in T_{u} U$, we get that

$$
\begin{aligned}
\left.\left(p_{2}^{*} \alpha\right)\right|_{u}\left(v^{(1)}, \ldots, v^{(k)}\right) & =\left.\alpha\right|_{p_{2}(u)}\left(\xi_{2}^{(1)}, \ldots, \xi_{2}^{(k)}\right) \quad \text { where } \xi_{2}^{(j)}:=\left(p_{2}\right)_{*} v^{(j)} \\
& =\left.\alpha\right|_{p_{2}(u)}\left(g \cdot \xi_{1}^{(1)}+\eta_{M}^{(1)}, \ldots, g \cdot \xi_{1}^{(k)}+\eta_{M}^{(k)}\right) \\
& \quad \text { where } \xi_{1}^{(j)}:=\left(p_{1}\right)_{*} v^{(j)} \text { and }\left.D_{v^{(j)}} a\right|_{u}=\eta^{(j)} \cdot g \\
& =\left.\alpha\right|_{p_{2}(u)}\left(g \cdot \xi_{1}^{(1)}, \ldots, g \cdot \xi_{1}^{(k)}\right) \quad \text { because } \alpha \text { is horizontal } \\
& =\left.\alpha\right|_{p_{1}(u)}\left(\xi_{1}^{(1)}, \ldots, \xi_{1}^{(k)}\right) \quad \text { because } \alpha \text { is invariant } \\
& =\left.\left(p_{1}^{*} \alpha\right)\right|_{u}\left(v^{(1)}, \ldots, v^{(k)}\right) .
\end{aligned}
$$

Unfortunately, in applying Proposition 2.20, it might be impossible to choose $g$ to be a smooth function of $u$ : 
Example 5.2 $\left(\mathbb{Z}_{2} \circlearrowright \mathbb{R}\right)$. Let $M=\mathbb{R}$, let $G=\{1,-1\}$ with $( \pm 1) \cdot x= \pm x$, and let $\pi: M \rightarrow M / G$ be the quotient map. Consider the two plots $p_{1}: \mathbb{R} \rightarrow M$ and $p_{2}: \mathbb{R} \rightarrow M$ defined as follows:

$$
p_{1}(t):= \begin{cases}-e^{-1 / t^{2}} & \text { if } t<0, \\ 0 & \text { if } t=0, \\ e^{-1 / t^{2}} & \text { if } t>0,\end{cases}
$$

and

$$
p_{2}(t):= \begin{cases}-e^{-1 / t^{2}} & \text { if } t \neq 0, \\ 0 & \text { if } t=0 .\end{cases}
$$

Then $\pi \circ p_{1}=\pi \circ p_{2}$. However, for $t<0$ we have $p_{1}(t)=1 \cdot p_{2}(t)$, whereas for $t>0$ we have $p_{1}(t)=-1 \cdot p_{2}(t)$, and so the two plots do not differ by a continuous function to $G$ on any neighbourhood of $t=0$.

Our proofs use the following lemma.

Lemma 5.3. Let $U \subseteq \mathbb{R}^{n}$ be an open set. Let $\left\{C_{i}\right\}$ be a (finite or) countable collection of relatively closed subsets of $U$ whose union is $U$. Then the union of the interiors, $\bigcup_{i} \operatorname{int}\left(C_{i}\right)$, is open and dense in $U$.

Proof. This is a consequence of the Baire category theorem.

We now prove Proposition 5.10 in the special case of a finite group action. In this case, basic differential forms are simply invariant differential forms, as the tangent space to an orbit at any point is trivial.

Proposition 5.4 (case of a finite group). Let $G$ be a finite group, acting on a manifold $M$. Then every basic form on $M$ is the pullback of a (diffeological) differential form on $M / G$.

Proof. Fix a basic differential $k$-form $\alpha$ on $M$. By Proposition 2.20, it is enough to show the following: if $p_{1}: U \rightarrow M$ and $p_{2}: U \rightarrow M$ are plots such that $\pi \circ p_{1}=\pi \circ p_{2}$, then $p_{1}^{*} \alpha=p_{2}^{*} \alpha$ on $U$. Fix two such plots $p_{1}: U \rightarrow M$ and $p_{2}: U \rightarrow M$. For each $g \in G$ let

$$
C_{g}:=\left\{u \in U \mid g \cdot p_{1}(u)=p_{2}(u)\right\} .
$$

By continuity, $C_{g}$ is closed for each $g$. By our assumption on $p_{1}$ and $p_{2}$,

$$
U=\bigcup_{g \in G} C_{g} .
$$

By Lemma 5.3, the set $\bigcup_{g \in G} \operatorname{int}\left(C_{g}\right)$ is open and dense in $U$. Thus, by continuity, it is enough to show that $p_{1}^{*} \alpha=p_{2}^{*} \alpha$ on $\operatorname{int}\left(C_{g}\right)$ for each $g \in G$. This, in turn, follows from the facts that, for each $g \in G$, we have $g \circ p_{1}=p_{2}$ on $\operatorname{int}\left(C_{g}\right)$ and $g^{*} \alpha=\alpha$.

Our next result, contained in Lemma 5.6 and preceded by Lemma 5.5, is a generalisation of the case of a finite group action: it shows that the property that interests us holds for a Lie group action if it holds for the action of the identity component of that Lie group, assuming that the action of the identity component is proper.

Lemma 5.5. Let $G$ be a Lie group, and let $G_{0}$ be its identity component. Assume that $G$ acts on a manifold $M$ such that the restricted action of $G_{0}$ on $M$ is proper. Then, for any $\gamma \in G / G_{0}$, and for any two plots $p_{1}: U \rightarrow M$ and $p_{2}: U \rightarrow M$, the set

$$
C_{\gamma}:=\left\{u \in U \mid \exists g \in \gamma \text { such that } g \cdot p_{1}(u)=p_{2}(u)\right\}
$$

is (relatively) closed in $U$. 
Proof. Because the $G_{0}$ action on $M$ is proper, the set

$$
\Delta:=\left\{\left(m, m^{\prime}\right) \in M \times M \mid \exists g_{0} \in G_{0} \text { such that } g_{0} \cdot m=m^{\prime}\right\}
$$

being the image of the proper map $\left(g_{0}, m\right) \mapsto\left(m, g_{0} \cdot m\right)$, is closed in $M \times M$.

Fix $g^{\prime} \in \gamma$. Because $G_{0}$ is normal in $G$, we can express $\gamma$ as the left coset $G_{0} g^{\prime}$, and we have

$$
C_{\gamma}=\left\{u \in U \mid \exists g_{0} \in G_{0} \text { such that } g_{0} g^{\prime} \cdot p_{1}(u)=p_{2}(u)\right\} \text {. }
$$

We conclude by noting that $C_{\gamma}$ is the preimage of the closed set $\Delta$ under the continuous map $U \rightarrow M \times M$ given by $u \mapsto\left(g^{\prime} \cdot p_{1}(u), p_{2}(u)\right)$.

Lemma 5.6. Let $G$ be a Lie group. Let $G_{0}$ be the identity component of $G$. Fix an action of $G$ on a manifold $M$. Suppose that the restricted $G_{0}$-action is proper, and suppose that every $G_{0}$-basic differential form on $M$ is the pullback of a diffeological form on $M / G_{0}$. Then every $G$-basic differential form on $M$ is the pullback of a diffeological form on $M / G$.

Proof. Fix a $G$-basic form $\alpha$ on $M$. Let $\pi: M \rightarrow M / G$ be the quotient map, and let $p_{1}: U \rightarrow M$ and $p_{2}: U \rightarrow M$ be plots such that $\pi \circ p_{1}=\pi \circ p_{2}$. Fix $\gamma \in G / G_{0}$ and $g^{\prime} \in \gamma$, and define $C_{\gamma}$ as in Lemma 5.5. Define $\tilde{p}_{1}: U \rightarrow M$ as the composition $g^{\prime} \circ p_{1}$. This is a plot of $M$, and for any $u \in \operatorname{int}\left(C_{\gamma}\right)$ we have $p_{2}(u)=g_{0} \cdot \tilde{p}_{1}(u)$ for some $g_{0} \in G_{0}$. Consider the restricted action of $G_{0}$ on $M$. Let $\pi_{0}: M \rightarrow M / G_{0}$ be the corresponding quotient map. Then the restrictions $\left.\tilde{p}_{1}\right|_{\operatorname{int}\left(C_{\gamma}\right)}$ and $\left.p_{2}\right|_{\operatorname{int}\left(C_{\gamma}\right)}$ are plots of $M$, and they satisfy $\left.\pi_{0} \circ \tilde{p}_{1}\right|_{\operatorname{int}\left(C_{\gamma}\right)}=\left.\pi_{0} \circ p_{2}\right|_{\operatorname{int}\left(C_{\gamma}\right)}$. By hypothesis, and because $\alpha$ is $G_{0}$-basic (as it is $G$-basic), $\alpha$ is a pullback of a diffeological form on $M / G_{0}$. By Proposition $2.20, \tilde{p}_{1}^{*} \alpha=p_{2}^{*} \alpha$ on $\operatorname{int}\left(C_{\gamma}\right)$.

But on $\operatorname{int}\left(C_{\gamma}\right)$ we have $\tilde{p}_{1}^{*} \alpha=p_{1}^{*} g^{\prime *} \alpha=p_{1}^{*} \alpha$ (since $\alpha$ is $G$-invariant), and so $p_{1}^{*} \alpha=p_{2}^{*} \alpha$ on $\operatorname{int}\left(C_{\gamma}\right)$. Since $\gamma \in G / G_{0}$ is arbitrary, and $\bigcup_{\gamma \in G / G_{0}} \operatorname{int}\left(C_{\gamma}\right)$ is open and dense in $U$ by Lemmas 5.3 and 5.5, from continuity we have that $p_{1}^{*} \alpha=p_{2}^{*} \alpha$ on all of $U$. Finally, by Proposition 2.20, $\alpha$ is the pullback of a form on $M / G$.

We proceed with two technical lemmas that we will use to handle non-trivial compact connected stabilisers.

Lemma 5.7. Let $G$ be a compact connected Lie group acting orthogonally on some Euclidean space $V=\mathbb{R}^{N}$. Let $g \in G$ and $\eta \in \mathfrak{g}$ be such that $\exp (\eta)=g$. Let $v \in V$. Then there exists $v^{\prime} \in V$ such that $\left|v^{\prime}\right| \leq|v|$ and $g \cdot v-v=\eta \cdot v^{\prime}$.

Proof. Since $V$ is a vector space, we identify tangent spaces at points of $V$ with $V$ itself. We also identify elements of the group $G$ and of the Lie algebra $\mathfrak{g}$ with the matrices by which these elements act on $V=\mathbb{R}^{N}$

$$
\begin{aligned}
g \cdot v-v & =\left.\exp (t \eta) \cdot v\right|_{0} ^{1} \\
& =\int_{0}^{1}\left(\frac{d}{d t} \exp (t \eta) \cdot v\right) d t \\
& =\int_{0}^{1}(\eta \cdot \exp (t \eta) \cdot v) d t \\
& =\eta \cdot \int_{0}^{1}(\exp (t \eta) \cdot v) d t
\end{aligned}
$$


So define $v^{\prime}:=\int_{0}^{1}(\exp (t \eta) \cdot v) d t$. Finally,

$$
\begin{aligned}
\left|v^{\prime}\right| & =\left|\int_{0}^{1}(\exp (t \eta) \cdot v) d t\right| \\
& \leq \int_{0}^{1}|\exp (t \eta) \cdot v| d t \\
& =\int_{0}^{1}|v| d t \quad \text { because the action is orthogonal } \\
& =|v| .
\end{aligned}
$$

This completes the proof.

Lemma 5.8. Let $G$ be a compact connected Lie group acting orthogonally on some Euclidean space $V=\mathbb{R}^{N}$. Let $\gamma_{1}$ and $\gamma_{2}$ be smooth curves from $\mathbb{R}$ into $V$ such that $\gamma_{1}(0)=\gamma_{2}(0)=0$ and such that for every $t \in \mathbb{R}$ there exists $g_{t} \in G$ satisfying $\gamma_{2}(t)=g_{t} \cdot \gamma_{1}(t)$. Let $\xi_{1}=\dot{\gamma}_{1}(0)$ and $\xi_{2}=\dot{\gamma}_{2}(0)$. Then, for every horizontal form $\alpha$ on $V$, we have $\left.\left(\xi_{2}-\xi_{1}\right)\right\lrcorner\left.\alpha\right|_{0}=0$.

Note that, in the assumptions of this lemma, $t \mapsto g_{t}$ is not necessarily continuous.

Proof. We claim that there exists a sequence of vectors $v_{n}^{\prime}$ converging to 0 in $V$, and a sequence $\mu_{n}$ in $\mathfrak{g}$, such that

$$
\xi_{2}-\xi_{1}=\lim _{n \rightarrow \infty} \mu_{n} \cdot v_{n}^{\prime}
$$

Indeed, choose any sequence of non-zero real numbers $t_{n}$ converging to 0 . For each $n$ choose $\eta_{n} \in \mathfrak{g}$ such that $\exp \left(\eta_{n}\right)=g_{t_{n}}$. Since we are working on a vector space, we can subtract the curves and consider $\gamma_{2}(t)-\gamma_{1}(t)$. We have

$$
\begin{aligned}
\xi_{2}-\xi_{1} & =\left.\frac{d}{d t}\right|_{t=0}\left(\gamma_{2}(t)-\gamma_{1}(t)\right) \\
& =\lim _{t \rightarrow 0}\left(\frac{\gamma_{2}(t)-\gamma_{1}(t)}{t}\right) \\
& =\lim _{n \rightarrow \infty}\left(\frac{\gamma_{2}\left(t_{n}\right)-\gamma_{1}\left(t_{n}\right)}{t_{n}}\right) \\
& =\lim _{n \rightarrow \infty}\left(\frac{g_{t_{n}} \cdot \gamma_{1}\left(t_{n}\right)-\gamma_{1}\left(t_{n}\right)}{t_{n}}\right) \\
& =\lim _{n \rightarrow \infty}\left(\frac{\eta_{n} \cdot v_{n}^{\prime}}{t_{n}}\right)
\end{aligned}
$$

for some $\eta_{n} \in \mathfrak{g}$ and for some $v_{n}^{\prime} \in V$ that satisfy $\left|v_{n}^{\prime}\right| \leq\left|\gamma_{1}\left(t_{n}\right)\right|$; the last equality is a result of Lemma 5.7. Because $\left|v_{n}^{\prime}\right| \leq\left|\gamma_{1}\left(t_{n}\right)\right| \underset{n \rightarrow \infty}{\rightarrow}\left|\gamma_{1}(0)\right|=0$, the claim holds with $\mu_{n}:=\eta_{n} / t_{n}$.

We now have

$$
\begin{aligned}
\left.\left(\xi_{2}-\xi_{1}\right)\right\lrcorner\left.\alpha\right|_{0} & \left.=\lim _{n \rightarrow \infty}\left(\left(\mu_{n} \cdot v_{n}^{\prime}\right)\right\lrcorner\left(\left.\alpha\right|_{v_{n}^{\prime}}\right)\right) \\
& \left.=\left.\lim _{n \rightarrow \infty}\left(\left(\mu_{n}\right)_{V}\right\lrcorner \alpha\right|_{v_{n}^{\prime}}\right),
\end{aligned}
$$

where $\left(\mu_{n}\right)_{V}$ is the vector field on $V$ induced by $\mu_{n} \in \mathfrak{g}$. Because $\alpha$ is horizontal, the last term above vanishes. 
The final ingredient that we need for Proposition 5.10 is the following property of the model that appears in the slice theorem (Theorem 2.25). Let $G$ be a Lie group, $H$ a closed subgroup, and $V$ a vector space with a linear $H$-action. Recall that the equivariant vector bundle $G \times_{H} V$ over $G / H$ is obtained as the quotient of $G \times V$ by the anti-diagonal $H$-action $h \cdot(g, v)=$ $\left(g h^{-1}, h \cdot v\right)$ and that the $G$-action on $G \times_{H} V$ is $g \cdot\left[g^{\prime}, v\right]=\left[g g^{\prime}, v\right]$.

Lemma 5.9. Suppose that every $H$-basic form on $V$ is the pullback of a diffeological form on $V / H$. Then every $G$-basic form on $G \times_{H} V$ is the pullback of a diffeological form on $\left(G \times{ }_{H} V\right) / G$.

Proof. Let $G \times H$ act on $G \times V$ where $G$ acts by left multiplication on the first factor and where $H$ acts by the anti-diagonal action $h:(g, v) \mapsto\left(g h^{-1}, h \cdot v\right)$. We have two maps:

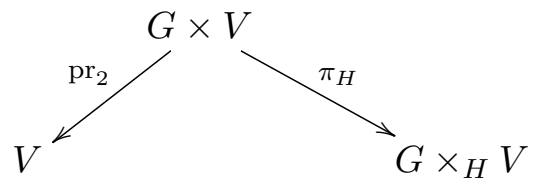

Here, the map $\pi_{H}: G \times V \rightarrow G \times_{H} V$ is the quotient by the $H$-action, and the projection to the second factor $\operatorname{pr}_{2}: G \times V \rightarrow V$ can be identified with the quotient by the $G$-action.

We apply quotient in stages (Lemma 4.1) in two ways: taking the quotient by $G$ and then by $H$, and taking the quotient by $H$ and then by $G$. This gives the following commuting diagram:

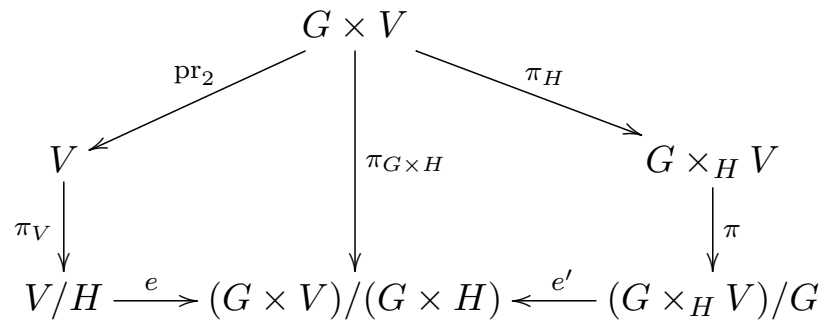

where $e$ and $e^{\prime}$ are diffeomorphisms, and $\pi_{V}, \pi_{G \times H}$, and $\pi$ are the quotient maps.

By hypothesis, the inclusion Image $\pi_{V}^{*} \subset \Omega_{\text {basic }}^{*}(V)$ is an equality. Applying Part (iv) of Lemma 4.1 to the left hand side of the diagram (5.1), we conclude that the inclusion Image $\pi_{G \times H}^{*} \subset \Omega_{\text {basic }}^{*}(G \times V)$ is an equality. Applying Part (iv) of Lemma 4.1 to the right hand side of the diagram (5.1), we further conclude that the inclusion Image $\pi^{*} \subset \Omega_{\mathrm{basic}}^{*}\left(G \times_{H} V\right)$ is an equality.

We are now ready to prove the main result of this section.

Proposition 5.10 (pullback surjects to basic forms). Let a Lie group $G$ act on a manifold $M$. Assume that the identity component of $G$ acts properly. Let $\pi: M \rightarrow M / G$ be the quotient map. Then every basic form on $M$ is the pullback of a (diffeological) differential form on $M / G$ via $\pi^{*}$.

Proof. By Lemma 5.6, to prove this result for an arbitrary Lie group, it is enough to prove it for the action of the identity component of the group.

For every non-negative integer $d$, consider the following two statements.

A(d): For every connected Lie group $K$ with $\operatorname{dim} K=d$, and for every $K$-manifold $N$ on which the $K$-action is proper, every $K$-basic form on $N$ is the pullback of a differential form on $N / K$.

$\mathrm{B}(\mathrm{d})$ : For every Lie group $K$ with $\operatorname{dim} K=d$, and for every $K$-manifold $N$ on which the identity component of $K$ acts properly, every $K$-basic form on $N$ is the pullback of a differential form on $N / K$. 
Since the result holds for the trivial group, Statement A(0) is true. By Lemma 5.6, it follows that Statement $\mathrm{B}(0)$ is true. Proceeding by induction, we fix a positive integer $d$, we assume that Statement $\mathrm{B}\left(\mathrm{d}^{\prime}\right)$ is true for all $d^{\prime}<d$, and we would like to prove that Statement $\mathrm{B}(\mathrm{d})$ is true. By Lemma 5.6, Statement A(d) implies Statement B(d); thus, it is enough to prove that Statement $A(d)$ is true. That is, we may now restrict to the special case of the proposition in which the group is connected and the action is proper, while assuming that the general case of the proposition is true for all Lie groups of smaller dimension.

Now, let $G$ be a connected Lie group, and let $M$ be a $G$-manifold on which the $G$-action is proper. Fix a $G$-basic form $\alpha$ on $M$. We would like to show that $\alpha$ is the pullback of a differential form on $M / G$.

By Proposition 2.20 we need to show, for any two plots $p_{1}: W \rightarrow M$ and $p_{2}: W \rightarrow M$ for which $\pi \circ p_{1}=\pi \circ p_{2}$, that $p_{1}^{*} \alpha=p_{2}^{*} \alpha$. Let $p_{1}$ and $p_{2}$ be two such plots. Fix $u \in W$. We would like to show that $\left.p_{1}^{*} \alpha\right|_{u}=\left.p_{2}^{*} \alpha\right|_{u}$.

Let $x=p_{2}(u)$. Let $H$ be the stabiliser of $x$. By Theorem 2.25 there exists a $G$-invariant open neighbourhood $U$ of $x$ and an equivariant diffeomorphism $F: U \rightarrow G \times{ }_{H} V$ where $V=$ $T_{x} M / T_{x}(G \cdot x)$. Because $F$ is an equivariant diffeomorphism and $\alpha$ is $G$-basic, $\left(F^{-1}\right)^{*} \alpha$ is $G$-basic on $G \times{ }_{H} V$.

Either $x$ is a fixed point, or $x$ is not a fixed point.

Suppose that $x$ is a fixed point. Then $H=G$. So $p_{1}(u)=p_{2}(u)=x$, and $F$ identifies $U$ with $V=T_{x} M$, sending $x$ to $0 \in V$. Fixing a $G$-invariant Riemannian metric, we have that $G$ acts linearly and orthogonally on $V$. Let $v \in T_{u} W$. Applying Lemma 5.8 to the curves $\gamma_{1}(t):=$ $F\left(p_{1}(u+t v)\right)$ and $\gamma_{2}(t):=F\left(p_{2}(u+t v)\right)$ in $V$ and to the basic form $\left(F^{-1}\right)^{*} \alpha$ on $V$, we obtain that $\left.\left.\dot{\gamma}_{1}(0)\right\lrcorner\left.\left(F^{-1}\right)^{*} \alpha\right|_{0}=\dot{\gamma}_{2}(0)\right\lrcorner\left.\left(F^{-1}\right)^{*} \alpha\right|_{0}$. This, in turn, implies that $\left.\left.v\right\lrcorner\left. p_{1}^{*} \alpha\right|_{u}=v\right\lrcorner\left. p_{2}^{*} \alpha\right|_{u}$. Because $v \in T_{u} W$ is arbitrary, we conclude that $\left.p_{1}^{*} \alpha\right|_{u}=\left.p_{2}^{*} \alpha\right|_{u}$, as required.

Suppose that $x$ is not a fixed point. Then the stabiliser $H$ of $x$ is a proper subgroup of $G$. Since $G$ is connected, $\operatorname{dim} H<\operatorname{dim} G$. By the induction hypothesis, every $H$-basic form on $V$ is the pullback of a differential form on $V / H$. By Lemma 5.9, every $G$-basic form on $G \times_{H} V$ is the pullback of a differential form on $\left(G \times_{H} V\right) / G$. Because $F$ is an equivariant diffeomorphism, every $G$-basic form on $U$ is the pullback of a differential form on $U / G$. So $\left.\alpha\right|_{U}$ is the pullback of a differential form on $U / G$. This implies that $\left.p_{1}^{*} \alpha\right|_{u}=\left.p_{2}^{*} \alpha\right|_{u}$, as required.

Proof of Theorem 1.1. By Lemma 3.1 and Proposition 3.2, the pullback is an injection into the space of basic forms. By Remark 2.17 and Proposition 3.4, as a map to its image, the pullback is an isomorphism of differential graded algebras and a diffeological diffeomorphism. By Proposition 5.10, if the identity component of $G$ acts properly, the image is the space of basic forms.

Example 5.11 (irrational torus, first construction). Fix an irrational number $\alpha \in \mathbb{R} \backslash \mathbb{Q}$. The corresponding irrational torus is

$$
T_{\alpha}:=\mathbb{R} /(\mathbb{Z}+\alpha \mathbb{Z}) .
$$

It is obtained as the quotient of $\mathbb{R}$ by the $\mathbb{Z}^{2}$-action $(m, n) \cdot x=x+m+n \alpha$; note that it is not Hausdorff. The basic differential forms on $\mathbb{R}$ with respect to this action are the constant functions and the constant coefficient one-forms $c d x$. By Proposition 5.10, each of these is the pullback of a differential form on $T_{\alpha}$.

Remark 5.12. In Example 5.11, although the topology of $T_{\alpha}$ is trivial, its de Rham cohomology is isomorphic to that of a circle. We note, though, that differential forms still do not capture the richness of the diffeology on $T_{\alpha}$ : by Donato and Iglesias [8], $T_{\alpha}$ and $T_{\beta}$ are diffeomorphic if and only if there exist integers $a, b, c, d$ such that $a d-b c= \pm 1$ and $\alpha=\frac{a+\beta b}{c+\beta d}$. See Exercise 4 and Exercise 105 of [14] with solutions at the end of the book. 
Example 5.13 (irrational torus, second construction). Fix an irrational number $\alpha \in \mathbb{R} \backslash \mathbb{Q}$. Consider the quotient

$\mathbb{T}^{2} / S_{\alpha}$

of $\mathbb{T}^{2}:=\mathbb{R}^{2} / \mathbb{Z}^{2}$ by the irrational solenoid $S_{\alpha}:=\{[t, \alpha t] \mid t \in \mathbb{R}\} \subset \mathbb{T}^{2}$. It is obtained as the quotient of $\mathbb{T}^{2}$ by the $\mathbb{R}$-action $t \cdot[x, y]=[x+t, y+\alpha t]$. The basic forms on $\mathbb{T}^{2}$ with respect to this action are the constant functions and the constant multiples of the one-form $\alpha d x-d y$. The quotient $\mathbb{T}^{2} / S_{\alpha}$ is diffeomorphic to the irrational torus $T_{\alpha}$ of Example 5.11; see Exercise 31 of [14] (with solution at the end of the book).

In fact, consider the action of $\mathbb{R} \times \mathbb{Z}^{2}$ on $\mathbb{R}^{2}$ that is given by $(t, m, n) \cdot(x, y)=(x+m+t, y+n+$ $\alpha t$ ). Taking the quotient first by $\mathbb{R}$ and then by $\mathbb{Z}^{2}$ (and identifying the first of these quotients with $\mathbb{R}$ through the map $(x, y) \mapsto y-\alpha x)$ yields $T_{\alpha}$. Taking the quotient first by $\mathbb{Z}^{2}$ and then by $\mathbb{R}$ yields $\mathbb{T}^{2} / S_{\alpha}$. Applying Lemma 4.1 twice, we get the following commuting diagram:

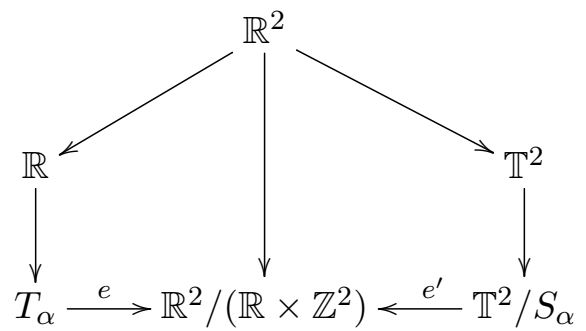

where $e$ and $e^{\prime}$ are diffeomorphisms. As noted in Example 5.11, every basic form on $\mathbb{R}$ is the pullback of a diffeological differential form on $T_{\alpha}$. By Lemma 4.1, this implies that every basic form on $\mathbb{R}^{2}$ is the pullback of a diffeological differential form on $\mathbb{R}^{2} /\left(\mathbb{R} \times \mathbb{Z}^{2}\right)$. Again by Lemma 4.1, we conclude that every basic form on $\mathbb{T}^{2}$ is the pullback of a diffeological form on $\mathbb{T}^{2} / S_{\alpha}$. Thus, the $\mathbb{R}$-action on $\mathbb{T}^{2}$ through $S_{\alpha}$ satisfies the conclusion of Proposition 5.10, although it does not satisfy the assumption of Proposition 5.10: this $\mathbb{R}$-action is not proper.

\section{A Orbifolds}

Let $X$ be a Hausdorff, second countable topological space. Fix a positive integer $n$.

The following definition is based on Haefliger, [11, Section 4].

1. An $n$ dimensional orbifold chart on $X$ is a triple $(\tilde{U}, \Gamma, \phi)$ where $\tilde{U} \subseteq \mathbb{R}^{n}$ is an open ball, $\Gamma$ is a finite group of diffeomorphisms of $\tilde{U}$, and $\phi: \tilde{U} \rightarrow X$ is a $\Gamma$-invariant map onto an open subset $U$ of $X$ that induces a homeomorphism $\tilde{U} / \Gamma \rightarrow U$.

2. Two orbifold charts on $X,(\tilde{U}, \Gamma, \phi)$ and $\left(\tilde{V}, \Gamma^{\prime}, \psi\right)$, are compatible if for every two points $u \in \tilde{U}$ and $v \in \tilde{V}$ such that $\phi(u)=\psi(v)$ there exist a neighbourhood $O_{u}$ of $u$ in $\tilde{U}$ and a neighbourhood $O_{v}$ of $v$ in $\tilde{V}$ and a diffeomorphism $g: O_{u} \rightarrow O_{v}$ that takes $u$ to $v$ and such that $\psi \circ g=\phi$.

3. An orbifold atlas on $X$ is a set of orbifold charts on $X$ that are pairwise compatible and whose images cover $X$. Two orbifold atlases are equivalent if their union is an orbifold atlas.

The following definition was introduced in [13]: A diffeological orbifold is a diffeological space that is locally diffeomorphic to finite linear quotients of $\mathbb{R}^{n}$.

These two definitions are equivalent in the following sense. Given an orbifold atlas on $X$, there exists a unique diffeology on $X$ such that all the homeomorphisms $\tilde{U} / \Gamma \rightarrow U$ are diffeomorphisms. With this diffeology, $X$ becomes a diffeological orbifold. Two orbifold atlases are 
equivalent if and only if the corresponding diffeologies are the same. Finally, every diffeological orbifold structure on $X$ can be obtained in this way. For details, see [13, Section 8].

Orbifolds were initially introduced by Ichiro Satake $[20,21]$ under the name "V-manifolds". Satake's approach is equivalent to Haefliger's; see [13]. Satake [21] also introduced tensors, and in particular differential forms, on V-manifolds. Haefliger's approach yields the following definition.

Let $\{(\widetilde{U}, \Gamma, \psi)\}$ be an orbifold atlas on $X$. An orbifold differential form on $X$ is given by, for each chart $(\widetilde{U}, \Gamma, \psi)$ in the atlas, a $\Gamma$-invariant differential form $\alpha_{\widetilde{U}}$ on the domain $\widetilde{U}$ of the chart. We require the following compatibility condition. For every two charts $(\widetilde{U}, \Gamma, \phi),\left(\tilde{V}, \Gamma^{\prime}, \psi\right)$, and every two points $u \in \widetilde{U}$ and $v \in \tilde{V}$ with $\phi(u)=\psi(v)$, there exist a diffeomorphism $g: O_{u} \rightarrow O_{v}$ from a neighbourhood of $u$ to a neighbourhood of $v$ that takes $u$ to $v$, such that $\psi \circ g=\phi$, and such that $g^{*}\left(\left.\alpha_{\tilde{V}}\right|_{O_{v}}\right)=\left.\alpha_{\widetilde{U}}\right|_{O_{u}}$. Two such collections $\left\{\alpha_{\widetilde{U}}\right\}$ of differential forms, defined on the domains of the charts in two equivalent orbifold atlases, represent the same orbifold differential form if their union still satisfies the compatibility condition.

Every diffeological differential form $\alpha$ on $X$ determines an orbifold differential form by associating to every chart $(\widetilde{U}, \Gamma, \psi)$ the pullback $\psi^{*} \alpha$. Proposition 5.4 implies that this gives a bijection between diffeological differential forms and orbifold differential forms.

\section{B Sjamaar differential forms; case of regular symplectic quotients}

Let a Lie group $G$ act properly on a symplectic manifold $(M, \omega)$ with an (equivariant) momentum map $\Phi: M \rightarrow \mathfrak{g}^{*}$. Let $Z=\Phi^{-1}(0)$ be the zero level set and $i: Z \rightarrow M$ its inclusion map. Let

$$
\begin{array}{r}
Z_{\text {reg }}=\left\{z \in Z \mid \exists \text { neighbourhood } U \text { of } z \text { in } Z \text { such that, for all } z^{\prime} \in U,\right. \\
\text { the stabilisers of } \left.z^{\prime} \text { and of } z \text { are conjugate in } G\right\} .
\end{array}
$$

The set $Z_{\text {reg}}$, (with the subset diffeology induced from $M$ or, equivalently, from $Z$ ) is a manifold, and it is open and dense in $Z$ (see [24]). The quotient $Z_{\mathrm{reg}} / G$, (with the quotient diffeology induced from $Z_{\text {reg }}$, or, equivalently, the subset diffeology induced from $M / G$,) is also a manifold.

Above, the connected components of $Z_{\mathrm{reg}}$ and $Z_{\mathrm{reg}} / G$ may have different dimensions. If $M$ is connected and $\Phi$ is proper, then $Z_{\mathrm{reg}}$ and $Z_{\mathrm{reg}} / G$ are connected. See, for example, [18] and [24].

Denote by $i_{\text {reg }}: Z_{\text {reg }} \rightarrow M$ the inclusion map and by $\pi_{\text {reg }}: Z_{\text {reg }} \rightarrow Z_{\text {reg }} / G$ the quotient map.

The following definition was introduced (but not yet named) by Reyer Sjamaar in [23]:

Definition B.1. A Sjamaar differential $l$-form $\sigma$ on $Z / G$ is a differential $l$-form on $Z_{\mathrm{reg}} / G$ (in the ordinary sense) such that there exists $\tilde{\sigma} \in \Omega^{l}(M)$ satisfying $i_{\text {reg }}^{*} \tilde{\sigma}=\pi_{\text {reg }}^{*} \sigma$.

A special case of a Sjamaar form is the reduced symplectic form, $\omega_{\text {red }}$, which satisfies $\pi_{\text {reg }}^{*} \omega_{\text {red }}=$ $i_{\text {reg }}^{*} \omega$. The orbit type stratification on $M$ induces a stratification of the reduced space $Z / G$, and the Sjamaar differential forms naturally extend to the strata of $Z / G$. The extensions of $\omega_{\text {red }}$ to these strata exhibit $Z / G$ as a stratified symplectic space in the sense of Sjamaar and Lerman [24].

The space of Sjamaar forms is closed under wedge products and forms a subcomplex of the de Rham complex $\left(\Omega^{*}\left(Z_{\mathrm{reg}} / G\right), d\right)$. Sjamaar forms satisfy a Poincaré lemma, Stokes' theorem, and a de Rham theorem.

For details, see Sjamaar's paper [23].

The reduced space $Z / G$ comes equipped with the quotient diffeology inherited from $Z$, which equals the subset diffeology inherited from $M / G$. We call this the subquotient diffeology.

It is now natural to ask how Sjamaar forms on a symplectic quotient $Z / G$, which a-priori depend on the ambient symplectic manifold $M$, relate to the diffeological forms on $Z / G$, whose 
definition is intrinsic. More precisely, consider the inclusion map $J: Z_{\text {reg }} / G \rightarrow Z / G$. Then we have the pullback map on diffeological forms

$$
J^{*}: \Omega^{l}(Z / G) \rightarrow \Omega^{l}\left(Z_{\mathrm{reg}} / G\right),
$$

and we identify the target space with the ordinary differential forms on $Z_{\text {reg }} / G$. We ask:

- Is the space of Sjamaar forms contained in the image of $J^{*}$ ?

- Is the image of $J^{*}$ contained in the space of Sjamaar forms?

- Is $J^{*}$ one-to-one?

If 0 is a regular value of the momentum map $\Phi$, then it follows from Proposition 5.4 that the answers to each of these questions is "yes". If 0 is a critical value, then the answer to the first question is "yes", and we do not know the answers to the other two questions. We refer the reader to Section 3.4 of the second author's thesis [28] for details.

\section{Acknowledgements}

This work is partially supported by the Natural Sciences and Engineering Council of Canada. We are grateful to Patrick Iglesias-Zemmour for instructing us on diffeology and to Reyer Sjamaar for his inspiration, as well as to the anonymous referees for excellent suggestions that lead to a better organisation of the paper.

\section{References}

[1] Baez J.C., Hoffnung A.E., Convenient categories of smooth spaces, Trans. Amer. Math. Soc. 363 (2011), 5789-5825, arXiv:0807.1704.

[2] Blohmann C., Fernandes M.C.B., Weinstein A., Groupoid symmetry and constraints in general relativity, Commun. Contemp. Math. 15 (2013), 1250061, 25 pages, arXiv:1003.2857.

[3] Bredon G.E., Introduction to compact transformation groups, Pure and Applied Mathematics, Vol. 46, Academic Press, New York - London, 1972.

[4] Chen K.-T., Iterated integrals of differential forms and loop space homology, Ann. of Math. 97 (1973), $217-246$.

[5] Chen K.-T., On differentiable spaces, in Categories in Continuum Physics (Buffalo, N.Y., 1982), Lecture Notes in Math., Vol. 1174, Springer, Berlin, 1986, 38-42.

[6] Crainic M., Struchiner I., On the linearization theorem for proper Lie groupoids, Ann. Sci. Éc. Norm. Supér. (4) 46 (2013), 723-746, arXiv:1103.5245.

[7] Cushman R., Śniatycki J., Differential structure of orbit spaces, Canad. J. Math. 53 (2001), 715-755.

[8] Donato P., Iglésias P., Exemples de groupes difféologiques: flots irrationnels sur le tore, C. R. Acad. Sci. Paris Sér. I Math. 301 (1985), 127-130.

[9] Duistermaat J.J., Kolk J.A.C., Lie groups, Universitext, Springer-Verlag, Berlin, 2000.

[10] Guillemin V., Ginzburg V., Karshon Y., Moment maps, cobordisms, and Hamiltonian group actions, Mathematical Surveys and Monographs, Vol. 98, Amer. Math. Soc., Providence, RI, 2002.

[11] Haefliger A., Groupoïdes d'holonomie et classifiants, Astérisque 116 (1984), 70-97.

[12] Hochschild G., The structure of Lie groups, Holden-Day, Inc., San Francisco - London - Amsterdam, 1965.

[13] Iglesias P., Karshon Y., Zadka M., Orbifolds as diffeologies, Trans. Amer. Math. Soc. 362 (2010), 2811-2831, math.DG/0501093.

[14] Iglesias-Zemmour P., Diffeology, Mathematical Surveys and Monographs, Vol. 185, Amer. Math. Soc., Providence, RI, 2013.

[15] Iglesias-Zemmour P., Karshon Y., Smooth Lie group actions are parametrized diffeological subgroups, Proc. Amer. Math. Soc. 140 (2012), 731-739, arXiv:1012.0107. 
[16] Karshon Y., Zoghi M., Orbifold groupoids and their underlying diffeology, Earlier version posted at http://www.math.toronto.edu/mzoghi/research/Groupoids.pdf and summarized in Zoghi's PhD thesis, University of Toronto, 2010.

[17] Koszul J.L., Sur certains groupes de transformations de Lie, in Géométrie différentielle (Colloques Internationaux du Centre National de la Recherche Scientifique, Strasbourg, 1953), Centre National de la Recherche Scientifique, Paris, 1953, 137-141.

[18] Lerman E., Meinrenken E., Tolman S., Woodward C., Nonabelian convexity by symplectic cuts, Topology 37 (1998), 245-259, dg-ga/9603015.

[19] Palais R.S., On the existence of slices for actions of non-compact Lie groups, Ann. of Math. 73 (1961), 295-323.

[20] Satake I., On a generalization of the notion of manifold, Proc. Nat. Acad. Sci. USA 42 (1956), 359-363.

[21] Satake I., The Gauss-Bonnet theorem for V-manifolds, J. Math. Soc. Japan 9 (1957), 464-492.

[22] Schwarz G.W., Smooth functions invariant under the action of a compact Lie group, Topology 14 (1975), 63-68.

[23] Sjamaar R., A de Rham theorem for symplectic quotients, Pacific J. Math. 220 (2005), 153-166, math.SG/0208080.

[24] Sjamaar R., Lerman E., Stratified symplectic spaces and reduction, Ann. of Math. 134 (1991), 375-422.

[25] Śniatycki J., Differential geometry of singular spaces and reduction of symmetry, New Mathematical Monographs, Vol. 23, Cambridge University Press, Cambridge, 2013.

[26] Souriau J.-M., Groupes différentiels, in Differential Geometrical Methods in Mathematical Physics (Proc. Conf., Aix-en-Provence/Salamanca, 1979), Lecture Notes in Math., Vol. 836, Springer, Berlin - New York, 1980, 91-128.

[27] Watts J., The calculus on subcartesian spaces, M.Sc. Thesis, University of Calgary, Canada, 2006.

[28] Watts J., Diffeologies, differential spaces, and symplectic geometry, Ph.D. Thesis, University of Toronto, Canada, 2012.

[29] Watts J., The orbit space and basic forms of a proper Lie groupoid, arXiv:1309.3001.

[30] Watts J., Wolbert S., Diffeology: a concrete foundation for stacks, arXiv:1406.1392. 